\title{
Latest Results on RPCs for the ATLAS LVL1 Muon Trigger
}

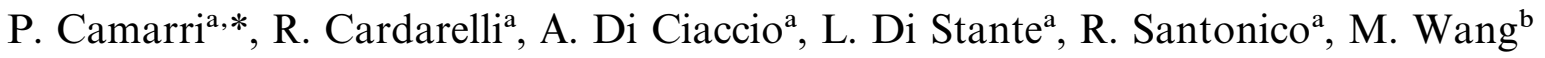 \\ a INFN Roma II and Dipartimento di Fisica dell'Università di Roma "Tor Vergata", Italy \\ ' IHEP Beijing, People's Republic of China
}

\begin{abstract}
The performances of 3, 2 and $1.5 \mathrm{~mm}$ gap RPCs operated with a heavy, environmentally safe industrial gas, $\mathrm{C}_{2} \mathrm{H}_{2} \mathrm{~F}_{4}$, have been tested at cosmic ray fluxes. Emphasis is given to the study of avalanche-to-streamer transition. (C) 1998 Elsevier Science B.V. All rights reserved.
\end{abstract}

Resistive Plate Chambers will be used as the LVL1 muon trigger detector in the barrel region $(|\eta|<1)$ of the ATLAS experiment at the LHC. Due to the high $\gamma$ and neutron background rate, RPCs will be operated in avalanche mode in order to provide higher rate capability. Owing to the low gas gain, external electronic amplification is needed before discriminating the readout strip signals. The RPC structure is described in detail elsewhere $[1,2]$.

We present here a test which was carried out at the Roma 2 INFN laboratories.

A cosmic ray trigger was provided by a signal coincidence of three scintillating slabs, parallel to the readout strips. We used a binary gas mixture: $\mathrm{C}_{2} \mathrm{H}_{2} \mathrm{~F}_{4} / \mathrm{C}_{4} \mathrm{H}_{10}=97 / 3$; gas mixtures containing $\mathrm{C}_{2} \mathrm{H}_{2} \mathrm{~F}_{4}$ were successfully used in a previous test [3]. Two-stage voltage amplifiers with an overall gain $\sim 250$ were used in the front end. Several gap widths were tested $(1.5,2$ and $3 \mathrm{~mm})$, and the following parameters were measured:

\footnotetext{
* Corresponding author.
}

- chamber efficiency, as a function of the operating voltage, for different discriminating thresholds;

- streamer fraction, as a function of the operating voltage, in order to study the avalanche-tostreamer transition and find the best operating conditions for RPCs (no front-end electronics was needed for this measurement);

- avalanche charge distributions.

With the purpose of taking into account the effects of temperature and pressure changes on the RPC operating point, a monitoring of these two quantities was performed and the voltage of each test was rescaled according to the rule $\mathrm{VT} / p=$ constant $(\mathrm{V}$ is the high voltage, $T$ the temperature in $\mathrm{K}$ and $p$ the pressure in mbar).

These measurements show that

- all the three gaps reach a very high plateau efficiency ( > 99\%);

- the efficiency plateau becomes from 100 to $200 \mathrm{~V}$ longer if the discriminating threshold is lowered from 100 to $30 \mathrm{mV}$ (at least for the 2 and $3 \mathrm{~mm}$ gaps) as shown in Fig. 1; 
- the avalanche charge distributions show a transition from a purely avalanche mode regime to a saturated one;

- the $3 \mathrm{~mm}$ gap avalanche/streamer separation is very narrow (Fig. 2), and the average streamer charge is very high with respect to the 1.5 and $2 \mathrm{~mm}$ gaps (Fig. 3).
Therefore, the use of $3 \mathrm{~mm}$ (and wider) gaps is not encouraged in connection with the gas mixture presently considered for the RPCs in ATLAS. Nevertheless, more gas mixtures will have to be tested in order to see if even better operating conditions exist.

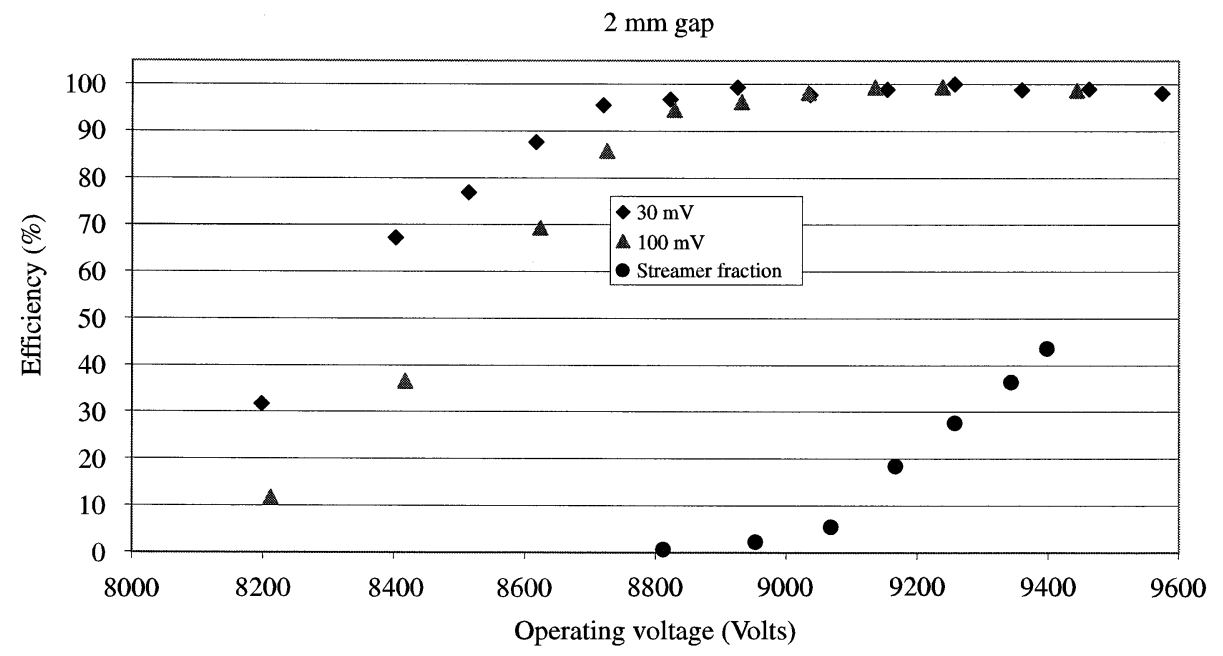

Fig. 1. Performance of the $2 \mathrm{~mm}$ gap RPC: the efficiency (for discriminating thresholds of $100 \mathrm{mV}$ and $30 \mathrm{mV}$ ) and the streamer fraction as a function of the operating voltage are shown.

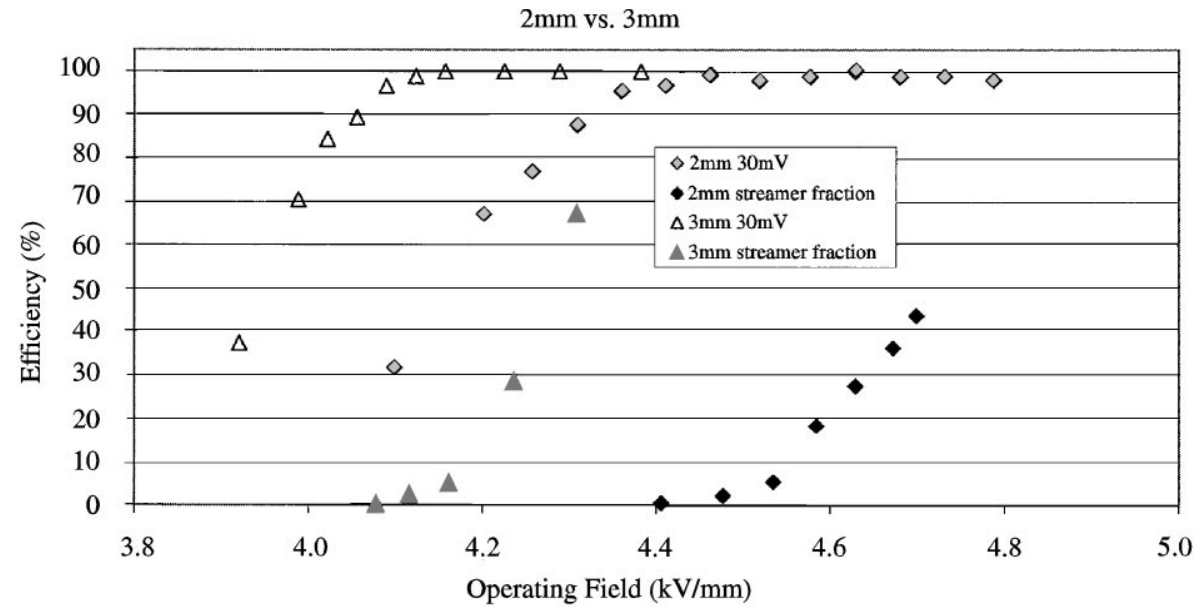

Fig. 2. Performances of 2 and $3 \mathrm{~mm}$ gap RPCs: the efficiency (for a discriminating threshold of $30 \mathrm{mV}$ ) and the streamer fraction as a function of the operating field are shown. 


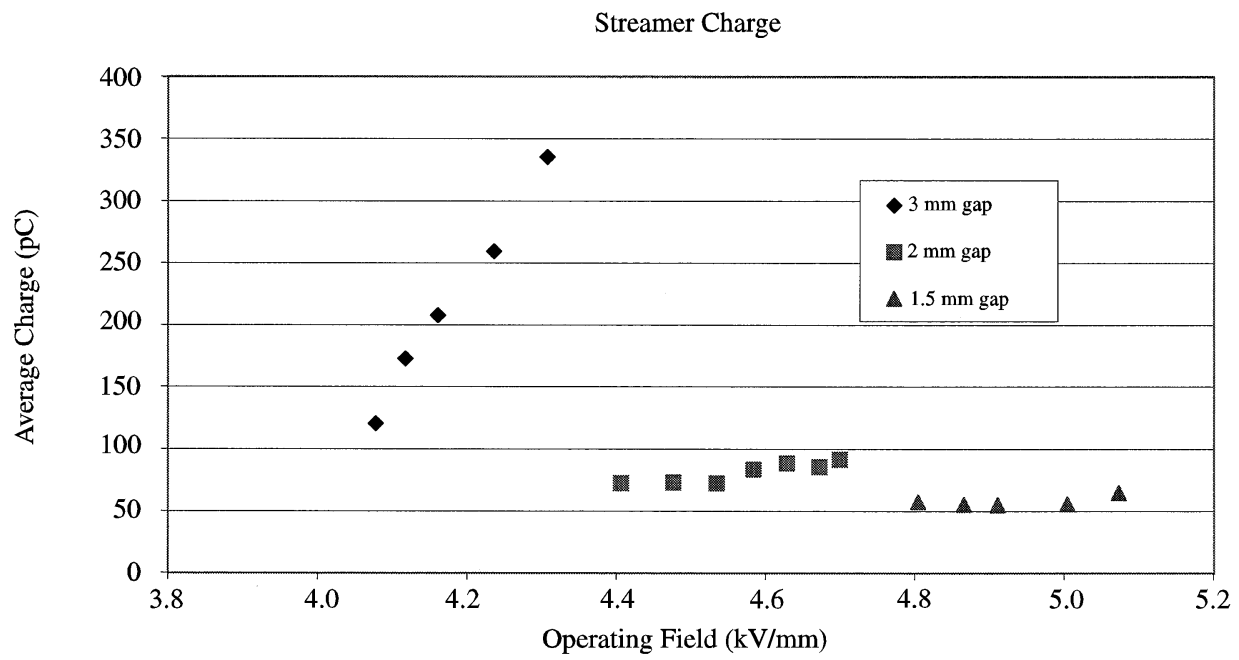

Fig. 3. Average streamer charge as a function of the operating field for the three RPCs under test.

\section{References}

[1] R. Santonico, R. Cardarelli, Nucl. Instr. and Meth. 187 (1981) 377.
[2] R. Cardarelli, R. Santonico, Nucl. Instr. and Meth. A 263 (1988) 200.

[3] R. Cardarelli et al., Nucl. Instr. and Meth. A 382 (1996) 470 . 